\title{
Can a one-layer optical skin model including melanin and inhomogeneously distributed blood explain spatially resolved diffuse reflectance spectra?
}

Hanna Karlsson, Anders Pettersson, Marcus Larsson and Tomas Strömberg

\section{Linköping University Post Print}

N.B.: When citing this work, cite the original article.

Original Publication:

Hanna Karlsson, Anders Pettersson, Marcus Larsson and Tomas Strömberg, Can a one-layer optical skin model including melanin and inhomogeneously distributed blood explain spatially resolved diffuse reflectance spectra?, 2011, Optical Tomography and Spectroscopy of Tissue IX, 78962Y.

http://dx.doi.org/10.1117/12.873134

Copyright: SPIE

Postprint available at: Linköping University Electronic Press

http://urn.kb.se/resolve?urn=urn:nbn:se:liu:diva-81240 


\title{
Can a one-layer optical skin model including melanin and inhomogeneously distributed blood explain spatially resolved diffuse reflectance spectra?
}

\author{
Hanna Karlsson ${ }^{a}$, Anders Pettersson $^{b}$, Marcus Larsson $^{a}$, Tomas Strömberg $^{a}$ \\ ${ }^{a}$ Department of Biomedical Engineering, Division of Biomedical Instrumentation, \\ Linköping University, S-581 85 Linköping, Sweden; \\ ${ }^{b}$ Perimed AB, Datavägen 9A, S-175 43 Järfälla, Stockholm, Sweden
}

\begin{abstract}
Model based analysis of calibrated diffuse reflectance spectroscopy can be used for determining oxygenation and concentration of skin chromophores. This study aimed at assessing the effect of including melanin in addition to hemoglobin $(\mathrm{Hb})$ as chromophores and compensating for inhomogeneously distributed blood (vessel packaging), in a single-layer skin model. Spectra from four humans were collected during different provocations using a twochannel fiber optic probe with source-detector separations 0.4 and $1.2 \mathrm{~mm}$. Absolute calibrated spectra using data from either a single distance or both distances were analyzed using inverse Monte Carlo for light transport and Levenberg-Marquardt for non-linear fitting. The model fitting was excellent using a single distance. However, the estimated model failed to explain spectra from the other distance. The two-distance model did not fit the data well at either distance. Model fitting was significantly improved including melanin and vessel packaging. The most prominent effect when fitting data from the larger separation compared to the smaller separation was a different light scattering decay with wavelength, while the tissue fraction of $\mathrm{Hb}$ and saturation were similar. For modeling spectra at both distances, we propose using either a multi-layer skin model or a more advanced model for the scattering phase function.
\end{abstract}

Keywords: diffuse reflectance spectroscopy, light transport, Monte Carlo simulation, tissue modeling, vessel packaging, skin

\section{INTRODUCTION}

Diffuse reflectance spectroscopy (DRS) using small source-detector distances, can be used for determining oxygenation and concentration of chromophores in tissue. ${ }^{1-4}$ Recordings may be done at a single source-detector distance $^{2,4,5}$ or at several distances. ${ }^{3,6,7}$ As for the modeling of light transport, empirical analytical models have been used ${ }^{2,4,5}$ or inverse models based on Monte Carlo simulations of light transport. ${ }^{3,6,7}$ The calibration may be done on a single optical phantom or using a set of phantoms. ${ }^{2,4,5}$ With a calibrated DRS system, absolute concentrations of chromophores can be determined. The choice of chromophores included in the absorption model is important. In myocardial spectra, the inclusion of cytochrome in addition to $\mathrm{Hb}$, lowered tissue fraction of $\mathrm{Hb}\left(f_{\mathrm{Hb}}\right)$ to about $50 \% .{ }^{8}$ Another aspect is whether the chromophores are diffusely found in a layer (melanin) or concentrated in vessels (hemoglobin ${ }^{8,9}$ ). In the myocardium, modeling blood in vessels rather than diffusely in tissue increased $f_{\mathrm{Hb}}$ by more than $250 \%$. Hence, modeling calibrated DRS concerns at least the following aspects: 1) which chromophores should be used; 2) how is light transport recorded and modeled; and 3) the tissue geometry, a single homogenous layer or a multilayer structure. ${ }^{10}$ In this work we used calibrated DRS with a single-layer geometry of the skin. The aim was to evaluate the effect of i) including melanin in the absorption model, ii) compensating for hemoglobin being located in vessels rather than homogenously in the tissue and iii) estimating tissue parameters from spectra recorded at two different source-detector distances.

Further author information: (Send correspondence to Hanna Karlsson) E-mail: hanna.karlsson@liu.se; Telephone: +46132867 50; Fax: +46 13101902 


\section{MATERIAL AND METHODS}

\subsection{Subjects}

After given informed consent, data were collected from four healthy, non-smoking human subjects (one male and three females), age 19 to 20. All subjects had Caucasian skin and had no disease or prescribed medication affecting the circulatory system. The subjects were asked to refrain from coffee for $2 \mathrm{~h}$ prior to the study. This study was approved by the Regional Ethical Review Board for Medical Research in Linköping (D. no M83-09).

\subsection{Equipment}

Diffuse reflectance spectra were recorded using a fiber optic probe connected to a multi-channel spectrometer (AvaSpec 2048-5-RM, Avantes BV, The Netherlands, grating: VB 600 lines/mm). The probe contained one emitting fiber, connected to a broadband white light source (Avalight-HAL-S, Avantes BV, The Netherlands), and two detector fibers at source-detector separations of $\rho=0.4$ and $1.2 \mathrm{~mm}$ (diameter: $200 \mu \mathrm{m}$; numerical aperture: 0.37; material: fused silica), each connected to a separate spectrometer channel. Variations in the lamp intensity were logged by a separate spectroscopic channel using an optical fiber directly connected to the lamp (denoted $\rho=0.0 \mathrm{~mm}$ ). To have a stable skin temperature and to ensure good tissue contact, the probe was placed in a thermostatic probe holder (PF 450, Perimed AB, Järfälla, Sweden).

\subsection{Measurement Protocol}

The measurements were performed in a room with an ambient temperature of $24.6 \pm 0.8^{\circ} \mathrm{C}$ where the subjects were lying comfortably with their arms and legs fully rested during the entire protocol. Three different provocations were conducted: local heating, systolic occlusion and venous occlusion. During local heating, the probe was placed on the dorsum of the foot. For the systolic and venous occlusion, the probe was placed on the volar area of the lower forearms.

All provocations were preceded by a 5 minutes baseline recording where the probe holder was heated to $32^{\circ} \mathrm{C}$. During the heat provocation the temperature was increased to $44^{\circ} \mathrm{C}$ and kept at that temperature for 25 minutes. For the remaining provocations, the probe holder was kept at a constant temperature of $32^{\circ} \mathrm{C}$. The systolic occlusion included a 5 minute suprasystolic pressure of $200 \mathrm{mmHg}$ of the upper arm using a cuff, followed by an immediate release off the cuff where the reperfusion phase was recorded for 5 additional minutes. Similarly, the venous occlusion consisted of a 5 minutes pressure above the venous pressure $(60 \mathrm{mmHg})$ of the upper arm followed by a 5 minute reperfusion phase.

\subsection{Calibration of the System}

After each measurement occasion a dark spectrum $M_{\text {dark }}\left(\rho, \lambda_{\text {raw }}, t\right)$ was recorded, time-averaged and finally subtracted from all measured spectra by calculating

$$
M^{\mathrm{d}}\left(\rho, \lambda_{\text {raw }}, t\right)=M\left(\rho, \lambda_{\text {raw }}, t\right)-\left\langle M_{\text {dark }}\left(\rho, \lambda_{\text {raw }}, t\right)\right\rangle_{t} .
$$

The dark corrected spectra were smoothed using a zero-phase low-pass filter with a filter kernel width of 2.5 $\mathrm{nm}$ (full-width half-maximum). Before normalizing each spectra with the detected lamp spectra (see Equation 2 ), data was resampled in order to remove variations in the channel specific wavelengths where intensities are recorded. The resampled spectra are denoted $M^{\mathrm{d}}(\rho, \lambda, t)$. Any changes in lamp intensity or color were then removed from the measured spectra by

$$
M^{\mathrm{ld}}(\rho, \lambda, t)=\frac{M^{\mathrm{d}}(\rho, \lambda, t)}{M^{\mathrm{d}}(0, \lambda, t)}
$$

All other calibration measurements were time-average before being used in the following calibration procedure. To quantify device dependent differences between measurement occasions, caused by e.g. reconnecting the optical fibers, a measurement on a custom made intensity reference phantom was performed at each occasion. All measured spectra were later normalized by the intensity reference spectra $\left(M_{\mathrm{intref}}^{\mathrm{ld}}(\rho, \lambda)\right)$ from the same occasion. The spectral characteristic of the intensity reference phantom was determined at a single separate 
calibration occasion $\left(t_{C}\right)$ by comparing it's reflectance, $M_{\text {intref, } \text { tC }_{\mathrm{C}}}^{\mathrm{ld}}(\rho, \lambda)$, with the reflectance from a surface covered with barium sulphate, $M_{\mathrm{BaSO}_{4}, \mathrm{t}_{\mathrm{C}}}^{\mathrm{ld}}(\rho, \lambda)$. The ratio between these two measurements was used for normalizing measurements conducted at a different occasion, i.e.

$$
M^{\mathrm{ild}}(\rho, \lambda, t)=\frac{M^{\mathrm{ld}}(\rho, \lambda, t)}{M_{\text {intref }}^{\mathrm{ld}}(\rho, \lambda)} \cdot \frac{M_{\mathrm{intref}, \mathrm{t}_{\mathrm{C}}}^{\mathrm{ld}}(\rho, \lambda)}{M_{\mathrm{BaSO}_{4}, \mathrm{t}_{\mathrm{C}}}^{\mathrm{ld}}(\rho, \lambda)} .
$$

Any intensity variation between the channels was managed by calibration factors, $k(\rho)$, for each fiber $(\rho=$ $0.4,1.2 \mathrm{~mm})$. These factors were calculated from a recording at the calibration occasion, $M_{\mathrm{ui}, \mathrm{t}_{\mathrm{C}}}^{\mathrm{d}}(\rho, \lambda)$, where all fibers were uniformly illuminated using an integrating sphere connected to an external light source, i.e.

$$
k(\rho)=\left\langle\frac{M_{\mathrm{ui}, \mathrm{t}_{\mathrm{C}}}^{\mathrm{d}}(\rho, \lambda)}{M_{\mathrm{BaSO}_{4}, \mathrm{t}_{\mathrm{C}}}^{\mathrm{d}}(\rho, \lambda)} \cdot\left\langle\frac{M_{\mathrm{ui}, \mathrm{t}_{\mathrm{C}}}^{\mathrm{d}}(\rho, \lambda)}{M_{\mathrm{BaSO}_{4}, \mathrm{t}_{\mathrm{C}}}^{\mathrm{d}}(\rho, \lambda)}\right\rangle_{\rho}^{-1}\right\rangle_{\lambda}^{-1} .
$$

To link measured and Monte Carlo simulated spectra, a recording on a well-defined polystyrene microsphere suspension (radius $155 \mu \mathrm{m}$ ) was made at the calibration occasion $\left(M_{\mu-\text { sphere, } \mathrm{t}_{\mathrm{C}}}^{\mathrm{ld}}(\rho, \lambda)\right)$. A single calibration factor was then calculated as

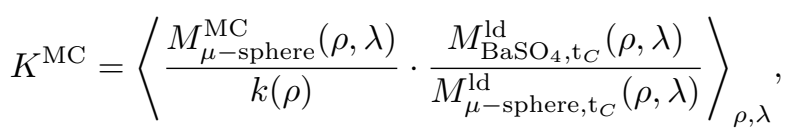

where $M_{\mu-\text { sphere }}^{\mathrm{MC}}(\rho, \lambda)$ denotes the Monte Carlo simulated spectra from the microsphere suspension. These spectra were simulated using a wavelength dependent Mie phase function, a scattering coefficient determined using a collimated transmission setup ${ }^{11}$ and the wavelength dependent absorption coefficient for water. ${ }^{12}$ The calibration procedure resulted in the absolute calibrated spectra

$$
T(\rho, \lambda, t)=K^{\mathrm{MC}} \cdot k(\rho) \cdot M_{\mathrm{tissue}}^{\mathrm{ild}}(\rho, \lambda, t) .
$$

\subsection{Data Analysis}

The absolute calibrated tissue spectra recorded during the different provocations, were time averaged in 10 second intervals. Four different phases were chosen: at the end of the baseline measurement before the heat provocation, at the end of the heat provocation, at the end of the systolic occlusion and at the end of the venous occlusion. Data were then analyzed using inverse Monte Carlo technique where a pre-simulated intensity matrix with different combinations of the absorption and the reduced scattering coefficient was compared to the measured spectra. In this analysis, the reduced scattering coefficient was assumed to decay with wavelength according to $\mu_{\mathrm{s}}^{\prime}(\lambda)=\alpha \cdot(\lambda / 650)^{-\beta}$, while the absorption coefficient $\mu_{\mathrm{a}}$ was modeled according to model I - IV below. The intensity matrix was simulated using the Henyey-Greenstein phase function with an anisotropy factor $\mathrm{g}=0.8$.

In model I, the tissue absorption coefficient was modeled as a linear combination of the oxygenized and reduced hemoglobin chromophores, $\mathrm{HbO}_{2}$ and $\mathrm{HbR}$ respectively, together with water. The expression for this model is given by

$$
\mu_{\mathrm{a}, \mathrm{I}}(\lambda)=f_{\mathrm{HbR}} \cdot \mu_{\mathrm{a}, \mathrm{HbR}}(\lambda)+f_{\mathrm{HbO}_{2}} \cdot \mu_{\mathrm{a}, \mathrm{HbO}_{2}}(\lambda)+f_{\mathrm{H}_{2} \mathrm{O}} \cdot \mu_{\mathrm{a}, \mathrm{H}_{2} \mathrm{O}}(\lambda),
$$

where $f_{\mathrm{i}}$ is the fraction of the chromophore $\mathrm{i}$. The total fraction of hemoglobin, $f_{\mathrm{HbR}}+f_{\mathrm{HbO}_{2}}$, is denoted $f_{\mathrm{Hb}}$. The fraction of water was assumed to be $0.75 .{ }^{13}$

In model II the chromophore melanin was included, resulting in the absorption expression

$$
\mu_{\mathrm{a}, \mathrm{II}}(\lambda)=f_{\mathrm{HbR}} \cdot \mu_{\mathrm{a}, \mathrm{HbR}}(\lambda)+f_{\mathrm{HbO}_{2}} \cdot \mu_{\mathrm{a}, \mathrm{HbO}_{2}}(\lambda)+f_{\mathrm{Mel}} \cdot \mu_{\mathrm{a}, \mathrm{Mel}}(\lambda)+f_{\mathrm{H}_{2} \mathrm{O}} \cdot \mu_{\mathrm{a}, \mathrm{H}_{2} \mathrm{O}}(\lambda) .
$$

In model III and IV, the effect of inhomogeneous blood distribution was taken into account by modifying the hemoglobin absorption spectra according to van Veen et al. ${ }^{9}$ This effect is implemented as the wavelength dependent amplification factor $\mathrm{C}$ using the expression

$$
C(\lambda)=\frac{1-\exp \left(-2 \cdot R \cdot 0.15 \cdot\left(s_{\mathrm{Hb}} \cdot \mu_{\mathrm{a}, \mathrm{HbO}_{2}}(\lambda)+\left(1-s_{\mathrm{Hb}}\right) \cdot \mu_{\mathrm{a}, \mathrm{HbR}}(\lambda)\right)\right)}{2 \cdot R \cdot 0.15 \cdot\left(s_{\mathrm{Hb}} \cdot \mu_{\mathrm{a}, \mathrm{HbO}_{2}}(\lambda)+\left(1-s_{\mathrm{Hb}}\right) \cdot \mu_{\mathrm{a}, \mathrm{HbR}}(\lambda)\right)},
$$


where $s_{\mathrm{Hb}}=f_{\mathrm{HbO}_{2}} /\left(f_{\mathrm{HbR}}+f_{\mathrm{HbO}_{2}}\right)$ is the tissue oxygenation and $R$ is the vessel radius. For model III and IV this results in the absorption models

$$
\mu_{\mathrm{a}, \mathrm{III}}(\lambda)=C(\lambda) \cdot\left(f_{\mathrm{HbR}} \cdot \mu_{\mathrm{a}, \mathrm{HbR}}(\lambda)+f_{\mathrm{HbO}_{2}} \cdot \mu_{\mathrm{a}, \mathrm{HbO}_{2}}(\lambda)\right)+f_{\mathrm{H}_{2} \mathrm{O}} \cdot \mu_{\mathrm{a}, \mathrm{H}_{2} \mathrm{O}}(\lambda)
$$

and

$$
\mu_{\mathrm{a}, \mathrm{IV}}(\lambda)=C(\lambda) \cdot\left(f_{\mathrm{HbR}} \cdot \mu_{\mathrm{a}, \mathrm{HbR}}(\lambda)+f_{\mathrm{HbO}_{2}} \cdot \mu_{\mathrm{a}, \mathrm{HbO}_{2}}(\lambda)\right)+f_{\mathrm{Mel}} \cdot \mu_{\mathrm{a}, \mathrm{Mel}}(\lambda)+f_{\mathrm{H}_{2} \mathrm{O}} \cdot \mu_{\mathrm{a}, \mathrm{H}_{2} \mathrm{O}}(\lambda) .
$$

The fitting parameters $\alpha, \beta, f_{\mathrm{HbR}}, f_{\mathrm{HbO}_{2}}, f_{\mathrm{Mel}}$ and $R$ were determinated for each model using the LevenbergMarquardt technique by minimizing the normalized residual spectra

$$
E(\rho, \lambda)=\frac{T^{\mathrm{MC}}\left(\mu_{\mathrm{a}}(\lambda), \mu_{\mathrm{s}}^{\prime}(\lambda), \rho\right)}{T(\rho, \lambda)}-1
$$

in a least-square sense, according to

$$
\min _{\alpha, \beta, f_{\mathrm{i}}, R}\left\|E(\rho, \lambda), \frac{\partial}{\partial \lambda} E(\rho, \lambda)\right\|
$$

where $T^{\mathrm{MC}}$ is the iteratively updated Monte Carlo simulated spectra. Absorption spectra for oxygenized and reduce hemoglobin were compiled from Zijlstra et al. ${ }^{14}$ Melanin absorption was approximated to $\mu_{\mathrm{a}, \mathrm{Mel}}=$ $1.7 \cdot 10^{11} \cdot \lambda^{-3.48}\left[\mathrm{~mm}^{-1}\right] .{ }^{15}$ The optimally fitted spectra (one for each of the four models) and the corresponding normalized residual spectra were denoted $T^{\mathrm{MC} *}$ and $E^{*}$, respectively. The fitting parameters $\alpha, \beta, f_{\mathrm{i}}$ and $R$ presented in the result and the discussion section all refer to the optimized parameters.

\subsection{Statistical Analysis}

The spectral fitting of the four models, I-IV, were statistically compared using the sum of the squared unnormalized residual spectra, $D^{*}=T^{\mathrm{MC} *}(\rho, \lambda)-T(\rho, \lambda)$. The effect of including melanin was tested using the test variable

$$
v=\left(\left(\left(D_{\mathrm{IV}}^{*}\right)^{2}-\left(D_{\mathrm{III}}^{*}\right)^{2}\right) /(6-5)\right) /\left(\left(D_{\mathrm{IV}}^{*}\right)^{2} /(55-6-1)\right),
$$

which is $\mathrm{F}(1,48)$-distributed.

The effect of vessel packaging was tested using

$$
\eta=\left(\left(\left(D_{\mathrm{IV}}^{*}\right)^{2}-\left(D_{\mathrm{II}}^{*}\right)^{2}\right) /(6-5)\right) /\left(\left(D_{\mathrm{IV}}^{*}\right)^{2} /(55-6-1)\right),
$$

which is $\mathrm{F}(1,48)$-distributed . A $v>7.3$ indicates that model IV has a significantly lower residual than model III at a level of significance of $\mathrm{p}<0.01$ and similarly for $\eta$ (critical value for $\mathrm{F}(1,40)$ ). This test comparing the residual spectra for different models is described in Kleinbaum et al. ${ }^{16}$

\section{RESULTS}

Data from fitting spectra recorded at a source-detector distance $\rho=0.4 \mathrm{~mm}$ are given for all models in Table 1. The comparison of residual spectra indicated that model IV had a significantly smaller residual than model III in 16 out of 16 spectra $(v>7.3)$ and that model IV had a significantly smaller residual than model II in 13 out of 16 spectra $(\eta>7.3)$. Model III gave a poor fit in the $520-600 \mathrm{~nm}$ band (Figure 1 ). The inclusion of melanin resulted in a lower $s_{\mathrm{Hb}}$ (model IV vs. model III, Table 1). The inclusion of vessel packaging gave a slightly higher $f_{\mathrm{Hb}}$ and $s_{\mathrm{Hb}}$ (model IV vs. model II, Table 1).

The data from fitting spectra recorded at a source-detector distance $\rho=1.2 \mathrm{~mm}$, are given in Table 2. The comparison of residual spectra indicated that model IV had a significantly smaller residual than model III in 16 out of 16 spectra $(v>7.3)$ and that model IV had a significantly smaller residual than model II in 16 out of 16 spectra $(\eta>7.3)$. In comparison with fitting at $\rho=0.4 \mathrm{~mm}$, fitting at $\rho=1.2 \mathrm{~mm}$ gave a higher $\beta$ (Table 2).

If fitting is simultaneously done at spectra recorded at the source-detector distances $\rho=0.4 \mathrm{~mm}$ and $\rho=1.2$ $\mathrm{mm}$, the average intensities for both channels are optimized while the spectral shape in the 520-600 nm region 


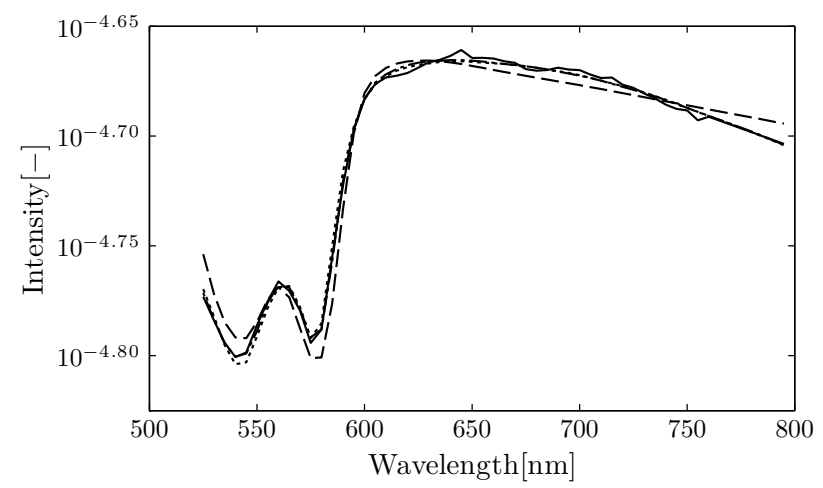

(a)

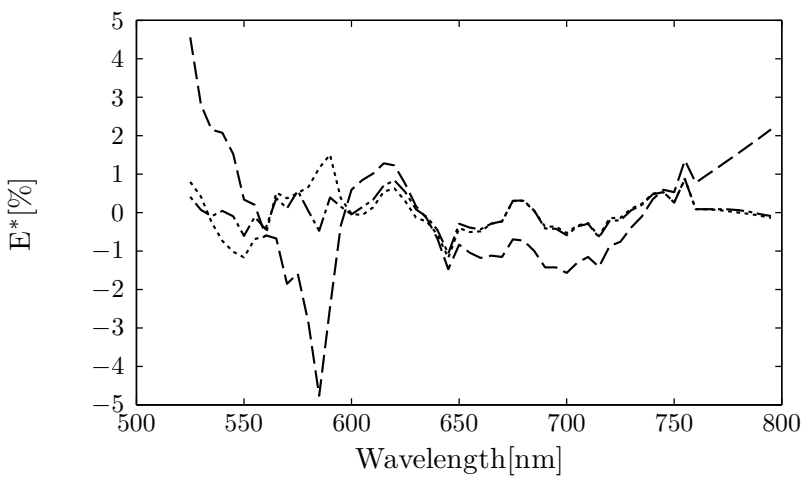

(c)

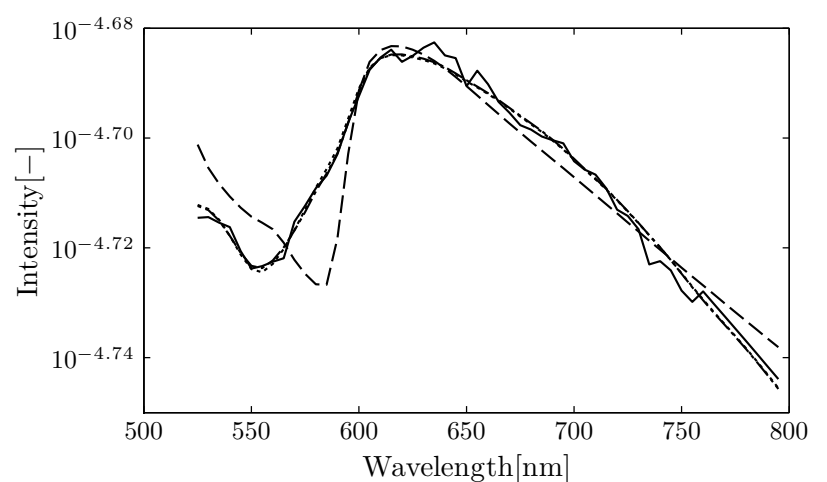

(b)

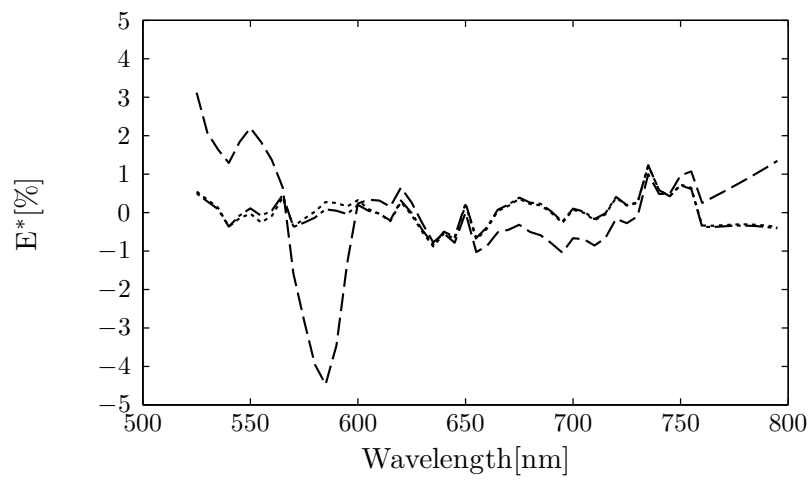

(d)

Figure 1. Absolute calibrated tissue spectrum $T(\rho, \lambda)$ (solid) and the fitted spectra at $\rho=0.4$ mm using model II (dotted), model III (dashed), and model IV (dash-dotted) for a representative recording (subject \#1). (a) At heat provocation (b) At systolic occlusion (c) Normalized residual spectra, heat provocation (d) Normalized residual spectra, systolic occlusion

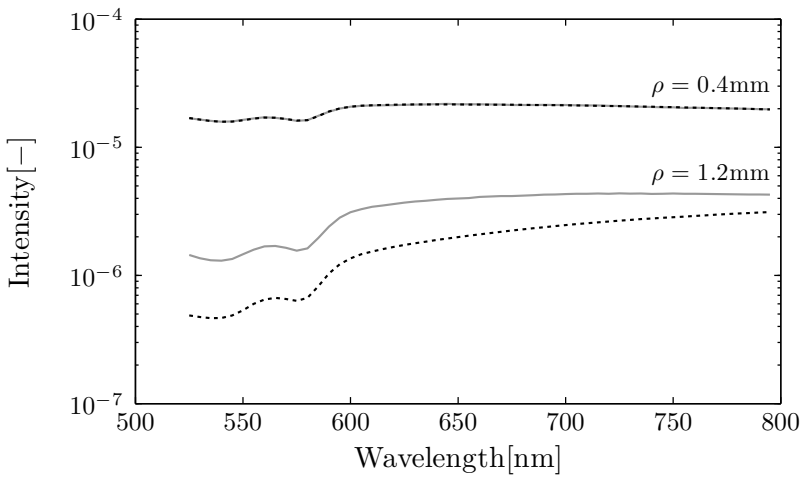

(a)

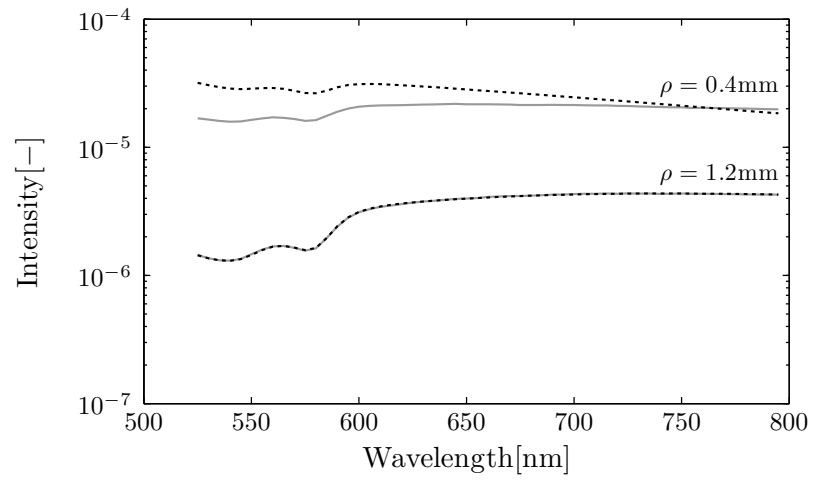

(b)

Figure 2. Absolute calibrated tissue spectrum $T(\rho, \lambda)$ (solid gray) and the fitted spectra using model IV (dotted black) for subject \#1 at heat provocation. (a) Fitting spectra at $\rho=0.4 \mathrm{~mm}$. (b) Fitting spectra at $\rho=1.2 \mathrm{~mm}$ 
Table 1. Tissue model parameters when fitting spectra recorded at $\rho=0.4 \mathrm{~mm}$. Data are given as M (SD), calculated from data from all four subjects, for $f_{\mathrm{Hb}}$ (the tissue fraction of $\mathrm{Hb}$ ), $s_{\mathrm{Hb}}$ (oxygen saturation), $f_{\mathrm{Mel}}$ (the tissue fraction of melanin), $\alpha$ (scattering modeling), $\beta$ (scattering modeling), $R$ (vessel radius), $\left\|E^{*}\right\|$ (model error).

\begin{tabular}{|l|l|l|l|l|l|}
\hline Model & Parameter & Baseline & Heat & Systolic occlusion & Venous occlusion \\
\hline I & $f_{\mathrm{Hb}}[\%]$ & $0.059(0.026)$ & $0.090(0.032)$ & $0.076(0.031)$ & $0.101(0.021)$ \\
\hline I & $s_{\mathrm{Hb}}[\%]$ & $54(5)$ & $71(5)$ & $12(7)$ & $21(6)$ \\
\hline I & $\alpha\left[\mathrm{mm}^{-1}\right]$ & $1.54(0.27)$ & $1.53(0.24)$ & $1.38(0.07)$ & $1.49(0.17)$ \\
\hline I & $\beta$ & $0.48(0.16)$ & $0.41(0.19)$ & $0.44(0.27)$ & $0.55(0.32)$ \\
\hline I & $\left\|E^{*}\right\|[\%]$ & $1.20(0.47)$ & $1.23(0.38)$ & $1.07(0.44)$ & $0.94(0.30)$ \\
\hline II & $f_{\mathrm{Hb}}[\%]$ & $0.069(0.037)$ & $0.114(0.042)$ & $0.094(0.044)$ & $0.125(0.029)$ \\
\hline II & $s_{\mathrm{Hb}}[\%]$ & $49(7)$ & $69(5)$ & $1(1)$ & $16(5)$ \\
\hline II & $f_{\mathrm{Mel}}[\%]$ & $0.679(0.363)$ & $0.745(0.317)$ & $0.637(0.376)$ & $0.476(0.285)$ \\
\hline II & $\alpha\left[\mathrm{mm}^{-1}\right]$ & $2.23(0.54)$ & $2.28(0.52)$ & $1.92(0.28)$ & $1.93(0.27)$ \\
\hline II & $\beta$ & $1.41(0.33)$ & $1.41(0.21)$ & $1.26(0.19)$ & $1.20(0.44)$ \\
\hline II & $\left\|E^{*}\right\|[\%]$ & $0.38(0.06)$ & $0.48(0.08)$ & $0.46(0.12)$ & $0.57(0.08)$ \\
\hline III & $f_{\mathrm{Hb}}[\%]$ & $0.127(0.056)$ & $0.153(0.043)$ & $0.182(0.085)$ & $0.169(0.019)$ \\
\hline III & $s_{\mathrm{Hb}}[\%]$ & $90(13)$ & $96(8)$ & $52(54)$ & $23(8)$ \\
\hline III & $\alpha\left[\mathrm{mm}^{-1}\right]$ & $1.54(0.27)$ & $1.53(0.24)$ & $1.39(0.07)$ & $1.51(0.18)$ \\
\hline III & $\beta$ & $0.50(0.15)$ & $0.42(0.19)$ & $0.47(0.31)$ & $0.64(0.30)$ \\
\hline III & $R[\mu \mathrm{m}]$ & $35.6(9.8)$ & $23.8(5.3)$ & $46.5(40.2)$ & $18.0(4.9)$ \\
\hline III & $\left\|E^{*}\right\|[\%]$ & $1.00(0.42)$ & $0.93(0.35)$ & $0.96(0.46)$ & $0.71(0.36)$ \\
\hline IV & $f_{\mathrm{Hb}}[\%]$ & $0.089(0.048)$ & $0.154(0.052)$ & $0.128(0.072)$ & $0.187(0.017)$ \\
\hline IV & $s_{\mathrm{Hb}}[\%]$ & $54(9)$ & $81(5)$ & $0(1)$ & $15(7)$ \\
\hline IV & $f_{\mathrm{Mel}}[\%]$ & $0.650(0.372)$ & $0.661(0.319)$ & $0.615(0.387)$ & $0.427(0.272)$ \\
\hline IV & $\alpha\left[\mathrm{mm}{ }^{-1}\right]$ & $2.21(0.55)$ & $2.20(0.50)$ & $1.92(0.28)$ & $1.91(0.27)$ \\
\hline IV & $\beta$ & $1.39(0.35)$ & $1.32(0.22)$ & $1.28(0.19)$ & $1.22(0.40)$ \\
\hline IV & $R[\mu \mathrm{m}]$ & $9.6(6.5)$ & $12.7(2.4)$ & $8.9(4.1)$ & $15.0(6.0)$ \\
\hline IV & $\left\|E^{*}\right\|[\%]$ & $0.33(0.04)$ & $0.34(0.04)$ & $0.34(0.04)$ & $0.32(0.06)$ \\
\hline
\end{tabular}

Table 2. Tissue model parameters when fitting spectra at $\rho=1.2 \mathrm{~mm}$. Data are given as M (SD).

\begin{tabular}{|l|l|l|l|l|l|}
\hline Model & Parameter & Baseline & Heat & Systolic occlusion & Venous occlusion \\
\hline IV & $f_{\mathrm{Hb}}[\%]$ & $0.073(0.025)$ & $0.163(0.043)$ & $0.114(0.061)$ & $0.229(0.108)$ \\
\hline IV & $s_{\mathrm{Hb}}[\%]$ & $57(12)$ & $82(5)$ & $0(0)$ & $21(10)$ \\
\hline IV & $f_{\mathrm{Mel}}[\%]$ & $0.208(0.048)$ & $0.249(0.072)$ & $0.314(0.052)$ & $0.220(0.138)$ \\
\hline IV & $\alpha\left[\mathrm{mm}^{-1}\right]$ & $2.29(0.31)$ & $2.18(0.25)$ & $1.72(0.28)$ & $1.96(0.49)$ \\
\hline IV & $\beta$ & $2.25(0.28)$ & $2.14(0.34)$ & $2.12(0.18)$ & $2.14(0.10)$ \\
\hline IV & $R[\mu \mathrm{m}]$ & $15.7(6.2)$ & $18.5(4.5)$ & $13.0(2.3)$ & $19.4(4.3)$ \\
\hline IV & $\left\|E^{*}\right\|[\%]$ & $0.36(0.03)$ & $0.50(0.15)$ & $0.44(0.08)$ & $0.44(0.07)$ \\
\hline
\end{tabular}


Table 3. Tissue model parameters when simultaneously fitting spectra recorded at $\rho=0.4 \mathrm{~mm}$ and $\rho=1.2 \mathrm{~mm}$. Data are given as $\mathrm{M}(\mathrm{SD})$.

\begin{tabular}{|l|l|l|l|l|l|}
\hline Model & Parameter & Baseline & Heat & Systolic occlusion & Venous occlusion \\
\hline IV & $f_{\mathrm{Hb}}[\%]$ & $0.179(0.060)$ & $0.238(0.013)$ & $0.406(0.223)$ & $0.217(0.044)$ \\
\hline IV & $s_{\mathrm{Hb}}[\%]$ & $78(16)$ & $89(10)$ & $97(5)$ & $30(6)$ \\
\hline IV & $f_{\mathrm{Mel}}[\%]$ & $0.181(0.052)$ & $0.239(0.066)$ & $0.354(0.079)$ & $0.254(0.182)$ \\
\hline IV & $\alpha\left[\mathrm{mm}^{-1}\right]$ & $1.77(0.31)$ & $1.82(0.27)$ & $1.67(0.13)$ & $1.76(0.14)$ \\
\hline IV & $\beta$ & $0.87(0.20)$ & $0.95(0.24)$ & $0.92(0.34)$ & $1.14(0.63)$ \\
\hline IV & $R[\mu \mathrm{m}]$ & $36.4(5.4)$ & $26.5(4.3)$ & $115.8(35.7)$ & $17.5(9.0)$ \\
\hline IV & $\left\|E^{*}\right\|[\%]$ & $2.09(0.85)$ & $1.99(0.47)$ & $2.76(0.89)$ & $2.07(0.24)$ \\
\hline
\end{tabular}

does not match, causing erroneous $s_{\mathrm{Hb}}$ at systolic occlusion. Consequently, the fitting error was substantially larger than using a single fiber. The $f_{\mathrm{Hb}}$ and $R$ are also highly varying between the recordings (Table 3 ).

The spectral model when fitting using either $\rho=0.4 \mathrm{~mm}$ or $\rho=1.2 \mathrm{~mm}$, but evaluated at both distances using the optimized parameters, is presented in Figure 2. It clearly shows that fitting at one distance gives a very good fit at that distance, but that the model does not fit data at the other distance.

\section{DISCUSSION}

When using model based data analysis for decomposing a diffuse reflectance spectrum, the choice of tissue model is of great importance. A missing chromophore does not only affect the residual spectra. It is also likely to affect the estimated amount of other included chromophores as the existing compounds will compensate for the missing absorber. Similarly, the estimated tissue scattering can be affected. In a previous study on myocardial tissue, the residual spectra showed a systematic deviations that correlated to the absorption spectrum of cytochrome aa3, when that chromophore was omitted. ${ }^{8}$ Adding the cytochrome absorption to that model resulted in a significant decrease in the residual spectra and a halved $\mathrm{Hb}$ fraction. In this study the inclusion of melanin significantly improved the residual spectra while decreasing the $\mathrm{Hb}$ fraction by $12 \%$ on average (comparing model III and model IV in Table 1).

In tissue, hemoglobin is normally confined within the blood vessels. This inhomogeneous distribution will cause an apparent decrease in the absorption coefficient. ${ }^{9}$ The decrease is especially pronounced for high fractions of $\mathrm{Hb}$ in the 520-600 $\mathrm{nm}$ range, where $\mathrm{Hb}$ is a strong absorber. When including this vessel packaging effect in our model, the estimated fraction of $\mathrm{Hb}$ increased by $37 \%$ on average (comparing model II and model IV in Table 1). The effect is even more pronounced when used in myocardial tissue models, where the estimated fractions have been reported to increase more than $250 \%$. This difference can be explained by the much lower Hb fraction in skin $(0.14 \%$, Table 1) than in the myocardium (5\%). With the appropriate model (model IV), the estimated vessel radius did not vary between recording phases, especially when fitting spectra from the separation $\rho=1.2$ $\mathrm{mm}$ (Table 1-2). The estimated vessel radii in this study agreed with those found by Rajaram et al. ${ }^{17}$ and Lindbergh et al. ${ }^{8}$

The inclusion of melanin absorption also affected the estimated scattering parameters. Most noticeable, the average $\beta$ increased from 0.51 to 1.30, while $\alpha$ increased from 1.65 to 2.06 (comparing model III and model IV in Table 1). One could argue that this increase is purely caused by a cross-talk between melanin absorption and scattering, as both models have a similar wavelength dependency. However, from the residual spectra, it is apparent that the inclusion of melanin substantially improves the ability for the model to mimic measurements from real skin. This ability is central in order to attain reliable estimates, especially when it comes to $\mathrm{Hb}$ saturation. If the model does not fit spectra well in the 520-600 nm wavelength range, the estimation of tissue oxygenation is likely to be very wrong. This is the case for model III (no melanin) at occlusion, which gave a $s_{\mathrm{Hb}}$ of $52 \%$ with a high between-subjects variability, when the other models were close to zero, which is to be expected. 
For model IV, the average estimated fraction of $\mathrm{Hb}$ was $0.14 \%$ using either $\rho=0.4 \mathrm{~mm}$ or $\rho=1.2 \mathrm{~mm}$. This is equivalent with a blood volume fraction of about $1 \%\left(f_{\mathrm{Hb}} / 0.150\right)$. Similar fractions were found by Rajaram et al. ${ }^{17}$ and Fredriksson et al. ${ }^{18}$ The estimated $\mathrm{Hb}$ saturation remained almost unaffected by the choice of $\rho$. In contrary, the melanin fraction decreased with increased $\rho$. This can be explained by a decreased influence from melanin, located in the most superficial skin layer, as the sampling volume increases with $\rho$.

A consistently higher $\beta$ was found when fitting spectra at the larger separation. Therefore, the spectral model estimated at one distance failed to explain the spectra at the other distance. Trying to fit spectra from both fiber separations at the same time failed, resulting in obviously erroneous results (Table 3). The reason for this could be that a single-layer skin model is too simple. Fredriksson et al. used a six-layer skin model for laser Doppler flowmetry ${ }^{18}$ that was later reduced to three layers. ${ }^{10}$ A first extension of the single-layer model in this study could be a two-layer model with a top epidermal layer including melanin, possibly with a different scattering than the underlying layer. Another extension of our model is to use a more advanced modeling of the scattering phase function. Bevilacqua et al. ${ }^{6}$ showed that for distances close to one reduced mean free path $\left(\frac{1}{\left(\mu_{a}+\mu_{s}^{\prime}\right)}\right)$, not only the anisotropy factor, but also higher order moments of the phase function influences light transport. In this study, $\mu_{s}^{\prime}$ at $650 \mathrm{~nm}$ was about 2 ( $\alpha$ in Tables 1-3). Hence, the spectra recorded at $\rho=0.4$ $\mathrm{mm}$ are influenced not only by $\mu_{s}^{\prime}$.

In conclusion, a one-layer model, including melanin and inhomogeneously distributed blood, can explain DRS spectra using a single-distance recording. However, it fails to explain multi-distance recordings. For an improved model, we suggest either a multi-layer model or a more advanced modeling of the scattering phase function.

\section{ACKNOWLEDGMENTS}

The study was financed by Perimed $\mathrm{AB}$ and Linköping University through the Center for Excellence NIMEDCBDP (Center for Biomedical Data Processing) and by VINNOVA and Perimed AB through the SamBIO research collaboration program between companies and academia within bioscience (VINNOVA D.no. 200800149) and by NovaMedtech, supported by the European Union Regional Development Fund.

\section{REFERENCES}

[1] Palmer, G. M., Zhu, C., Breslin, T. M., Xu, F., Gilchrist, K. W., and Ramanujam, N., "Monte Carlo-based inverse model for calculating tissue optical properties. Part II: Application to breast cancer diagnosis," Applied Optics 45(5), 1072-1078 (2006).

[2] Bargo, P. R., Prahl, S. A., Goodell, T. T., Sleven, R. A., Koval, G., Blair, G., and Jacques, S. L., "In vivo determination of optical properties of normal and tumor tissue with white light reflectance and an empirical light transport model during endoscopy," Journal of Biomedical Optics 10(3), 1-15 (2005).

[3] Thueler, P., Charvet, I., Bevilacqua, F., Ghislain, M. S., Ory, G., Marquet, P., Meda, P., Vermeulen, B., and Depeursinge, C., "In vivo endoscopic tissue diagnostics based on spectroscopic absorption, scattering, and phase function properties," Journal of Biomedical Optics 8(3), 495-503 (2003).

[4] Lindbergh, T., Larsson, M., Szabó, Z., Casimir-Ahn, H., and Strömberg, T., "Intramyocardial oxygen transport by quantitative diffuse reflectance spectroscopy in calves," Journal of Biomedical Optics 15(2), 027009 (2010).

[5] Häggblad, E., Lindbergh, T., Karlsson, M. G., Casimir-Ahn, H., Salerud, E. G., and Strömberg, T., "Myocardial tissue oxygenation estimated with calibrated diffuse reflectance spectroscopy during coronary artery bypass grafting," Journal of Biomedical Optics 13(5), 054030 (2008).

[6] Bevilacqua, F., Piguet, D., Marquet, P., Gross, J. D., Tromberg, B. J., and Depeursinge, C., "In vivo local determination of tissue optical properties: Applications to human brain," Applied Optics 38(22), 4939-4950 (1999).

[7] Dam, J. S., Pedersen, C. B., Dalgaard, T., Fabricius, P. E., Aruna, P., and Andersson-Engels, S., "Fiberoptic probe for noninvasive real-time determination of tissue optical properties at multiple wavelengths," Applied Optics 40(7), 1155-1164 (2001). 
[8] Lindbergh, T., Larsson, M., Szabó, Z., Casimir-Ahn, H., and Strömberg, T., "Improved model for myocardial diffuse reflectance spectra by including mitochondrial cytochrome aa3, methemoglobin, and inhomogenously distributed RBC," Journal of Biophotonics (2010). n/a. doi: 10.1002/jbio.201000048.

[9] Van Veen, R. L. P., Verkruysse, W., and Sterenborg, H. J. C. M., "Diffuse-reflectance spectroscopy from 500 to $1060 \mathrm{~nm}$ by correction for inhomogeneously distributed absorbers," Optics Letters 27(4), 246-248 (2002).

[10] Fredriksson, I., Larsson, M., and Strömberg, T., "Model-based quantitative laser Doppler flowmetry in skin," Journal of Biomedical Optics 15(5), 057002 (2010).

[11] Lindbergh, T., Fredriksson, I., Larsson, M., and Strömberg, T., "Spectral determination of a two-parametric phase function for polydispersive scattering liquids," Optics Express 17(3), 1610-1621 (2009).

[12] Buiteveld, H., Hakvoort, J. H. M., and Donze, M., "Optical properties of pure water," Ocean Optics XII 2258(1), 174-183, SPIE (1994).

[13] Jacques, S., Bargo, P., and Engelking, E., "Optical fiber reflectance spectroscopy." [Online] Available at: http://omlc.ogi.edu/news/oct03/saratov/index.htm [Accessed 15 December 2010].

[14] Zijlstra, W., Buursma, A., and Assendelft, O., [Visible and Near Infrared Absorption Spectra of Human and Animal Haemoglobin Determination and Application], ch. 2, VSP BV, Utrecht, Boston, Köln \& Tokyo (2000).

[15] Jacques, S., "Optical absorption of melanin." [Online] Available at: http://omlc.ogi.edu/spectra/melanin/mua.html [Accessed 15 December 2010].

[16] Kleinbaum, D., Kupper, L., Nizam, A., and Muller, K., [Applied Regression Analysis and Multivariable Methods], ch. 16-4, Cengage Learning, Inc, New York, USA (2007).

[17] Rajaram, N., Gopal, A., Zhang, X., and Tunnell, J., "Experimental validation of the effects of microvasculature pigment packaging on in vivo diffuse reflectance spectroscopy," Lasers in Surgery and Medicine 42(7), 680-688 (2010).

[18] Fredriksson, I., Larsson, M., and Strömberg, T., "Optical microcirculatory skin model: assessed by Monte Carlo simulations paired with in vivo laser Doppler flowmetry," Journal of Biomedical Optics 13(1), 014015 (2008). 